\title{
A Sequence Kernel Association Test for Dichotomous Traits in Family Samples under a Generalized Linear Mixed Model
}

\author{
Qi Yan ${ }^{a}$ Hemant K. Tiwari ${ }^{a}$ Nengjun $\mathrm{Yi}^{\mathrm{a}}$ Guimin Gao $^{\mathrm{b}}$ Kui Zhang ${ }^{\mathrm{a}}$ \\ Wan-Yu Lin ${ }^{c}$ Xiang-Yang Lou ${ }^{a}$ Xiangqin Cui ${ }^{a}$ Nianjun Liu $^{a}$ \\ a Department of Biostatistics, University of Alabama at Birmingham, Birmingham, Ala., and bepartment of \\ Biostatistics, School of Medicine, Virginia Commonwealth University, Richmond, Va., USA; ' Institute of Epidemiology \\ and Preventive Medicine, College of Public Health, National Taiwan University, Taipei, Taiwan
}

\section{Key Words}

Dichotomous traits . Family samples . Generalized linear

mixed model · Linear kernel function . Sequence data analyze family data with dichotomous phenotypes while handling genetic variants with the same or opposite directions of effects as well as any types of family relationships.

ㄷ) 2015 S. Karger AG, Basel

\section{Introduction}

With recent advances in high-throughput sequencing technology, significant progress has been made in identifying the association between genetic variants and complex diseases $[1,2]$. Such progress requires appropriate study designs and statistical methods. Genome-wide association studies (GWASs) have been widely used both for identifying common single-nucleotide polymorphisms (SNPs) associated with human diseases and for further understanding the genetic basis of complex diseases [3-7]. In a typical GWAS, hundreds or thousands of individuals are recruited, and a large number of genetic markers are genotyped for all of the subjects. The association between the trait and each of the genetic markers is usually tested one by one through single-marker association tests. As a useful complement to the single-marker test, gene-based (or, more generally, set-based) tests are becoming more attractive [8-10]. If there are multiple causative variants

\section{KARGER 125}

() 2015 S. Karger AG, Base

$0001-5652 / 15 / 0792-0060 \$ 39.50 / 0$

E-Mail karger@karger.com

www.karger.com/hhe
Nianjun Liu

Department of Biostatistics, University of Alabama at Birmingham, RPHB 420A

1665 University Boulevard

Birmingham, AL 35294 (USA)

E-Mail nliu@uab.edu 
which have small individual effects, a single-marker analysis may not identify those weak signals. On the other hand, set-based tests have higher power because they combine the effects of all SNPs in the set and thus may be able to detect small effects. In addition, set-based tests may have higher power in the presence of genetic heterogeneity. And finally, set-based approaches greatly reduce the burden of multiple testing in GWASs.

Many set-based approaches have been developed in recent years $[8,9,11-22]$. One popular test is the sequence kernel association test (SKAT) $[9,21,22]$, a flexible, computationally efficient, and regression-based approach. In SKAT, covariates can be easily incorporated into the model. In addition, predefined weights can be assigned to each SNP in the SNP set. This increases the power when prior information shows that certain types of markers may be associated with a trait. For example, a weight as a function of a minor allele frequency (MAF) that follows a beta density is proposed in Wu et al. [21], which assumes that rare genetic variants have larger effects on common diseases. Furthermore, the test statistic derived in SKAT follows a mixture of $\chi^{2}$ distributions. Thus, the $\mathrm{p}$ values can be computed analytically without permutation, leading to a significant improvement in computation.

Family-based designs have been widely used to study the association between diseases and genetic variants [23-26]. In GWASs with unrelated samples, a general linear model is usually used to investigate the association between quantitative phenotypes and genetic markers. However, a general linear model results in an inflated type I error rate when the family correlation is not appropriately handled. Thus, instead of a general linear model, a linear mixed model including a random effect is usually employed to deal with correlation between family samples. The covariance of random effects of all individuals can be expressed by a variance of polygenic effect and kinship matrix (a matrix of kinship coefficients, which are measures of degrees of genetic correlations between individuals). Linear mixed models have been commonly used in single-marker GWASs for family data [27, 28]. Recently, SKAT has been extended to be applicable for quantitative traits in family samples [29-31]. Furthermore, the extension to dichotomous traits in family samples is described in Ionita-Laza et al. [32], in which the algorithm follows a generalized linear model and incorporates laws of Mendelian transmission to calculate the genotype expectation conditional on parental genotypes. This method, however, ignores parental phenotypes and, therefore, may lose power because it does not fully use the data. In addition, this method cannot handle nonparental rela- tionships such as those between offspring, grandparentsgrandchildren, or uncles-nephews.

In this study, we aim to propose a method that can handle dichotomous traits in family samples. Our proposed method uses a kernel machine regression and can be viewed as a generalization of famSKAT [29]. Our new model, denoted as F-SKAT, is based on a generalized linear mixed-model framework that is more general and can be applied to a larger range of studies with different types of traits. We demonstrate in our simulation studies that the original SKAT has inflated type I error rates when applied to all family samples without consideration of their relationship. By contrast, our proposed F-SKAT has a correct type I error rate. Moreover, because it uses all family samples, F-SKAT is consistently more powerful than SKAT with the use of only unrelated individuals (founders) in the family data because SKAT can only use a subset of the samples for analysis in order to retain the correct type I error rate. This is also consistent with the simulation results for quantitative traits in Chen et al. [29]. The same observation was also made in other studies [33].

\section{Methods}

SKAT in a Generalized Linear Mixed-Model Framework

The model setup is presented in a manner very similar to that of Chen et al. [29], although for noncontinuous traits instead. We assume that the $n \times 1$ vector of trait $y$ follows a generalized linear mixed model. Now, the trait is no longer assumed to follow a normal distribution. The link function $h(\cdot)$ is used to map a linear combination of predictors for observation $i, \eta_{i}$, to the conditional mean of observation $i, \mu_{i}=E\left(y_{i} \mid u, \gamma\right)$, shown as

$$
h(\mu)=\eta=X \beta+G \gamma+Z u,
$$

where $X$ is an $n \times p$ covariate matrix, $\beta$ is a $p \times 1$ vector standing for fixed effects parameters (an intercept and $p-1$ covariates), $G$ is an $n \times q$ genotype matrix for $q$ genetic variants of interest, $\gamma$ is a $q \times 1$ vector for the random effects of variants, $Z$ is an $n \times k$ matrix for $k$ random effects, and $u$ is a $k \times 1$ vector for the random effect coefficients, which is added to the original SKAT model $[9,21]$. The random effect $\gamma_{j}$ is assumed to be normally distributed with mean 0 and variance $\tau W_{j}$ for variant $j$, so the null hypothesis being tested is $H_{0}: \gamma=0$ that is equivalent to testing $H_{0}: \tau=0$, which can be tested with a variance component score test [21] in the mixed model. Also assumed is that $u$ is normally distributed and uncorrelated with $\gamma$, as in

$$
\begin{aligned}
& \gamma \sim N(0, \tau W), \\
& u \sim N(0, K),
\end{aligned}
$$

where $W$ is a predefined $q \times q$ diagonal weight matrix for each variant and may use $\sqrt{ } w_{i}=\operatorname{Beta}\left(M A F_{i}, 1,25\right)$ as in SKAT [21], and $K$ is a $k \times k$ covariance matrix.

Following the same rationale as in the derivation of the SKAT and famSKAT score statistics [29, 34-36] (see online suppl. mate- 
rial for details, www.karger.com/doi/10.1159/000375409), we have the following test statistic for our new model

$$
Q=\left(y^{*}-X \hat{\beta}\right)^{\prime} \hat{\Sigma}^{-1} G W G^{\prime} \hat{\Sigma}^{-1}\left(y^{*}-X \hat{\beta}\right),
$$

where $y^{*}$ is the final working trait vector, $\hat{\beta}$ is the vector of estimated fixed effects of covariates under $H_{0}$, and $\hat{\Sigma}$ is the estimated variance-covariance matrix under $H_{0}$. The statistic $Q$ is a quadratic form of $\left(y^{*}-X \hat{\beta}\right)$ and follows a mixture of $\chi^{2}$ distributions [37] under $H_{0}$. The $\mathrm{p}$ values can be calculated by numerical algorithms such as the Davies method [38]. This generalized linear model framework is very general with many models as special cases depending on the data type of the phenotype. For example, this framework can be simplified for continuous and dichotomous traits in a population-based study (see online suppl. Appendix), which is the same as the models described in Wu et al. [21]. In addition, count traits can also be handled. Furthermore, longitudinal and family structures can be added in the model by manipulating the random effect term.

\section{SKAT for Dichotomous Traits in Family Samples}

Specifically, the above approach can be used for handling dichotomous traits in family samples such that $h(\cdot)$ is replaced with $\operatorname{logit}(\cdot)$ and $Z u$ is replaced with $\delta$ that is an $n \times 1$ vector for the random effects of family correlation. The dichotomous trait $y$ follows a generalized linear mixed model

$$
\operatorname{logit}(P(y=1))=\operatorname{logit}(\mu)=X \beta+G \gamma+\delta,
$$

where $X \beta$ and $G \gamma$ are the same as defined above. Again, the random effect vector $\gamma$ is assumed to follow a normal distribution with mean 0 and covariance matrix $\tau W$, so $H_{0}: \tau=0$, where $W$ is the predefined weight matrix and may use $\sqrt{ } w_{i}=\operatorname{Beta}\left(M A F_{i}, 1,25\right)$. In addition, $\delta$ follows a normal distribution with mean 0 and covariance matrix $\sigma_{\delta}^{2} \Phi$, where $\Phi$ is twice the $n \times n$ kinship matrix obtained from family information only.

\section{Simulation Study}

We simulated samples based on a collection of 10,000 haplotypes over a $200-\mathrm{kb}$ region generated by the calibrated coalescent model [39] with mimicked linkage disequilibrium structure of European ancestry. Almost all of the simulated variants are rare variants; 1,200 haplotypes were randomly selected as parents' haplotypes. The offspring haplotypes were generated by randomly transmitting 1 of the 2 haplotypes of the father and the mother. We generated 300 families with a father, mother, and at least 1 offspring in each family for a scenario of families with flexible numbers of offspring (sibship size range: 1-5; fig. 1a). For simplicity, we also considered the scenario of family trios by selecting a father, mother, and 1 offspring from the 300 families (fig. 1b). In addition, in order to simulate a complex pedigree, we randomly selected 400 haplotypes as grandparents' haplotypes and 400 haplotypes as independent parents' haplotypes. For the 3-generation family scenario, we generated 100 families with 2 grandparents, 2 independent parents, 2 dependent parents who are the offspring of the 2 grandparents, and 4 children (fig. 1c). Furthermore, 30 randomly selected SNPs with at least one copy of a minor allele appearing in the corresponding data set were used in the analysis. The genotype can be easily converted from the haplotype data, and we simulated 100 such genotype data sets in the analysis for each of the three scenarios.
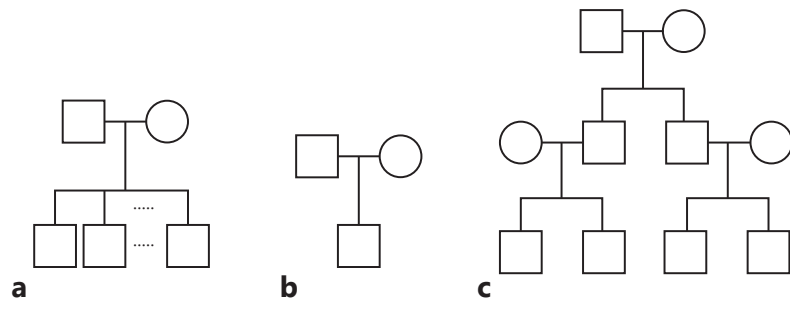

Fig. 1. Three scenarios of pedigree structures in simulation studies. a Families with flexible numbers of offspring. b Family trios. c Families with 3 generations.

\section{Type I Error Rate}

In analyzing the scenarios of families with flexible numbers of offspring and families with 3 generations, we compared F-SKAT to 3 other approaches: (1) the approach that applies SKAT on unrelated individuals (founders) in the family data (abbreviated as unrSKAT), (2) the original SKAT, and (3) the method by IonitaLaza et al. (abbreviated as IL) [32]. For each of the 100 genotype data sets, we simulated 1,000 sets of phenotypes. The dichotomous phenotypes for each family were generated via the following model

$$
\operatorname{logit}(P(y=1))=\alpha_{0}+\delta,
$$

where $\alpha_{0}$ was determined to set the prevalence to $10 \%$ in our simulation. In other words, $\alpha_{0}=\log (0.1 / 0.9)$, and $\delta$ is from a multivariate normal distribution with means 0 and covariance $\sum=\sigma_{\delta}^{2} \Phi$, where $\Phi$ is twice the kinship matrix. For instance, a family with a father, mother, and 2 children has

$$
\sum_{i}=\sigma_{\delta}^{2}\left[\begin{array}{cccc}
1 & 0 & 0.5 & 0.5 \\
0 & 1 & 0.5 & 0.5 \\
0.5 & 0.5 & 1 & 0.5 \\
0.5 & 0.5 & 0.5 & 1
\end{array}\right] \text {, }
$$

and $\sigma_{\delta}^{2}=5$. The scenario of families with 3 generations has a more complicated kinship matrix (online suppl. fig. 1S). The phenotypes for all of the families were generated in the same way, and the 1,000 simulated phenotypes for each of the 100 genotype data sets were used to evaluate the type I error rate. For the scenario of family trios, both parents and 1 child were selected from each of the families with flexible numbers of offspring. For these families, the covariance matrix $\sum_{i}$ is the same as for all the families, where

$$
\sum_{i}=\sigma_{\delta}^{2}\left[\begin{array}{ccc}
1 & 0 & 0.5 \\
0 & 1 & 0.5 \\
0.5 & 0.5 & 1
\end{array}\right] .
$$

\section{Power Evaluation}

We used the same genotype data sets as described above. We compared F-SKAT with unrSKAT and IL for the scenarios of families with flexible numbers of offspring and families with 3 generations. For each of the 100 genotype data sets, we simulated 1,000 
Table 1. Type I error rate of F-SKAT, unrSKAT, SKAT, and IL for parent-offspring family trios

\begin{tabular}{lllll}
\hline & $\alpha=0.05$ & $\alpha=0.01$ & $\alpha=0.005$ & $\alpha=0.001$ \\
\hline F-SKAT & 0.05188 & 0.01109 & 0.00577 & 0.00131 \\
unrSKAT & 0.04887 & 0.01009 & 0.00535 & 0.00138 \\
SKAT & 0.08802 & 0.02254 & 0.01239 & 0.0035 \\
IL & 0.05528 & 0.01380 & 0.00789 & 0.00195 \\
\hline
\end{tabular}

Table 2. Type I error rate of F-SKAT, unrSKAT, SKAT, and IL for families with flexible numbers of offspring

\begin{tabular}{lllll}
\hline & $\alpha=0.05$ & $\alpha=0.01$ & $\alpha=0.005$ & $\alpha=0.001$ \\
\hline F-SKAT & 0.05421 & 0.01151 & 0.00589 & 0.00134 \\
unrSKAT & 0.04887 & 0.01009 & 0.00535 & 0.00138 \\
SKAT & 0.12263 & 0.03530 & 0.02047 & 0.00598 \\
IL & 0.05242 & 0.01350 & 0.00766 & 0.00235 \\
\hline
\end{tabular}

Table 3. Type I error rate of F-SKAT, unrSKAT, SKAT, and IL for families with 3 generations

\begin{tabular}{lcccc}
\hline & $\alpha=0.05$ & $\alpha=0.01$ & $\alpha=0.005$ & $\alpha=0.001$ \\
\hline F-SKAT & 0.05356 & 0.01201 & 0.00625 & 0.00158 \\
unrSKAT & 0.04779 & 0.00989 & 0.00527 & 0.00142 \\
SKAT & 0.17455 & 0.05511 & 0.03308 & 0.01022 \\
IL & 0.05159 & 0.01269 & 0.00727 & 0.00189 \\
\hline
\end{tabular}

sets of phenotypes. The dichotomous phenotypes for each family were simulated via the following model

$$
\begin{aligned}
& \operatorname{logit}\left(P\left(y_{i}=1\right)\right)=\alpha_{0}+0.5 X_{1 i}+0.5 X_{2 i}+\beta_{1} G_{1 i}+\beta_{2} G_{2 i}+\ldots+ \\
& \beta_{k} G_{k i}+\delta_{i},
\end{aligned}
$$

where $X_{1 i}$ is a continuous variable generated from a standard normal distribution, $X_{2 i}$ is a dichotomous variable from a Bernoulli distribution with a probability of $0.5, G_{1 i}, G_{2 i}, \ldots, G_{k i}$ are the genotypes of causal SNPs, and $\beta_{1}, \beta_{2}, \ldots, \beta_{k}$ are log odds ratios of the causal SNPs. We considered that $40 \%$ and $80 \%$ of all variants are disease susceptibility variants and that $\alpha_{0}$ and $\delta_{i}$ were determined the same way as described in the Type I Error Rate section. Furthermore, $\beta_{1}, \beta_{2}, \ldots, \beta_{k}$ were set to $c \log _{10} M A F_{i}$ in order to assign large weights to rare variants, where $c=0.4$ is chosen such that when MAF $=0.0001, \beta=1.6$ (i.e., odds ratio $=4.9$ ) [21]. Because SKAT can handle the presence of both risk and protective variants, we also considered that $25 \%$ of the causal variants are protective, which means $\beta=-c \mid \log _{10} M A F_{i}$ I (i.e., $30 \%$ disease variants and $10 \%$ protective variants; and $60 \%$ disease variants and $20 \%$ protective variants). The phenotypes for all of the families were generated the same way, and the 1,000 phenotypes for each of the 100 genotype

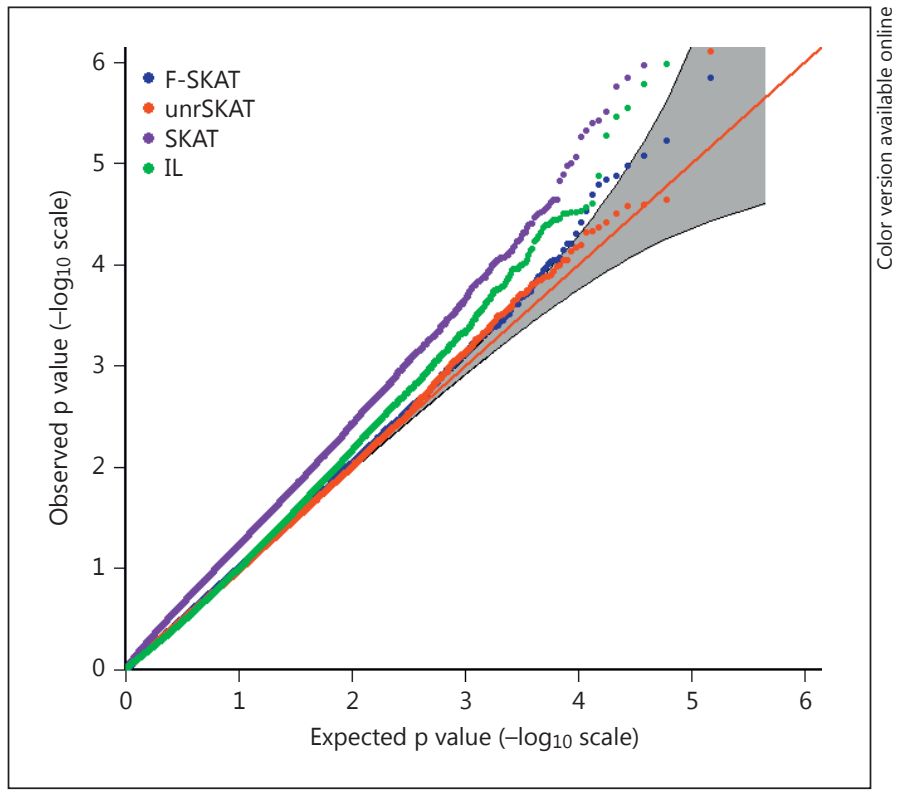

Fig. 2. QQ plot of the $\mathrm{p}$ values for F-SKAT, unrSKAT, SKAT, and IL for parent-offspring family trios from the null simulation, with a 95\% pointwise confidence band (gray area) that is computed under the assumption of the $p$ values being drawn independently from a uniform $[0,1]$ distribution.

data sets were used to evaluate the power. For the scenario of family trios, the simulated phenotypes of father, mother, and 1 offspring were selected from each of the families with flexible numbers of offspring.

\section{Results}

\section{Simulation of the Type I Error Rate}

Tables 1-3 depict the empirical type I error rates of FSKAT, unrSKAT, SKAT, and IL at a levels of 0.05, 0.01, 0.005 , and 0.001 for family trios, families with flexible numbers of offspring, and families with 3 generations. The results indicate that the type I error is inflated when SKAT is applied directly to all of the samples including correlated individuals. In contrast, F-SKAT, unrSKAT, and IL retain the correct type I error rate, but IL shows a trend of increased type I error rate as the significance level decreases. From the quantile-quantile (QQ) plots in figures 2 and 3, we can see similar patterns. This indicates that F-SKAT and unrSKAT can control type I error well for different significance levels; however, IL maintains the correct type I error rate when the significance level is not stringent (say, $\alpha>0.005$ ) but otherwise has an inflated type I error rate. The QQ plot in figure 4 indicates that 


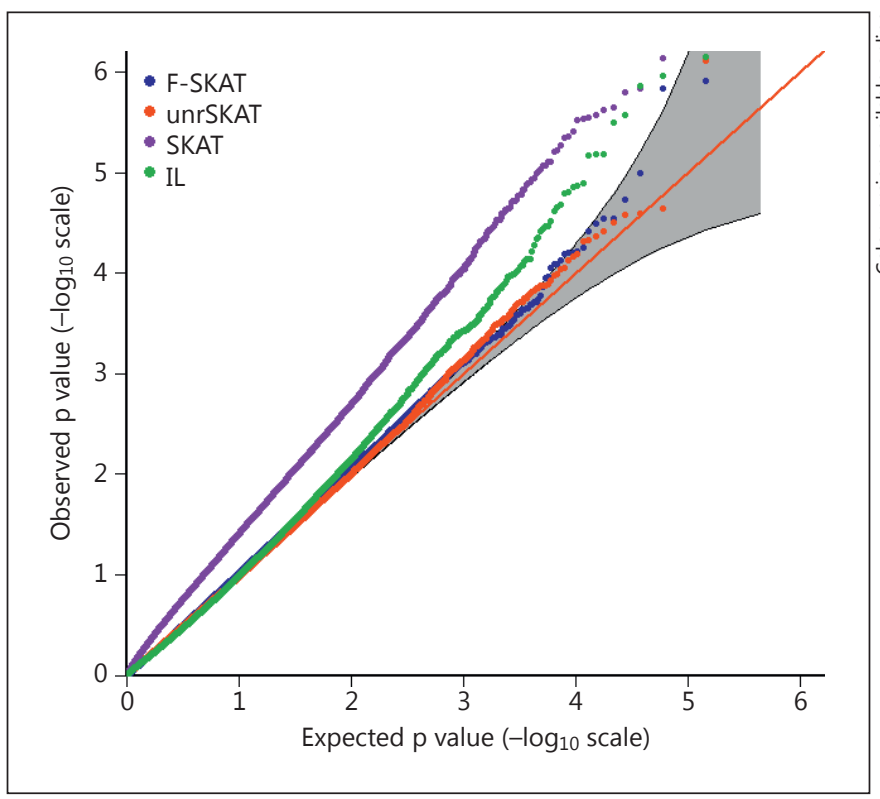

Fig. 3. QQ plot of the $\mathrm{p}$ values for F-SKAT, unrSKAT, SKAT, and IL for families with flexible numbers of offspring from the null simulation, with a $95 \%$ pointwise confidence band (gray area) that is computed under the assumption of the $\mathrm{p}$ values being drawn independently from a uniform $[0,1]$ distribution.

F-SKAT preserves the desired type I error rate, and unrSKAT and IL maintain the correct type I error rate at nonstringent significance levels. The inflation of the type I error rate in SKAT becomes more severe as the number of correlated individuals in one family increases.

\section{Statistical Power Comparison}

Because the original SKAT has an incorrect type I error rate for family data, we only investigated the power of F-SKAT, unrSKAT, and IL (i.e. exclude the original SKAT from the power comparison). The simulation results for family trios, families with flexible numbers of offspring, and families with 3 generations are shown in figures 5-7, respectively. For family trios, in all of the scenarios we considered, the power of F-SKAT is consistently but only slightly higher than that of unrSKAT. The slight gain in power may be caused by the difference in type I error rate (online suppl. fig. 2S). The power of IL is not high in our simulation study. For families with flexible numbers of offspring and families with 3 generations, the pattern is similar to that of the family trios. In all scenarios, the power of F-SKAT is consistently higher than that of unrSKAT and IL. Furthermore, F-SKAT achieves the highest power in families with 3 generations, and the power of F-SKAT

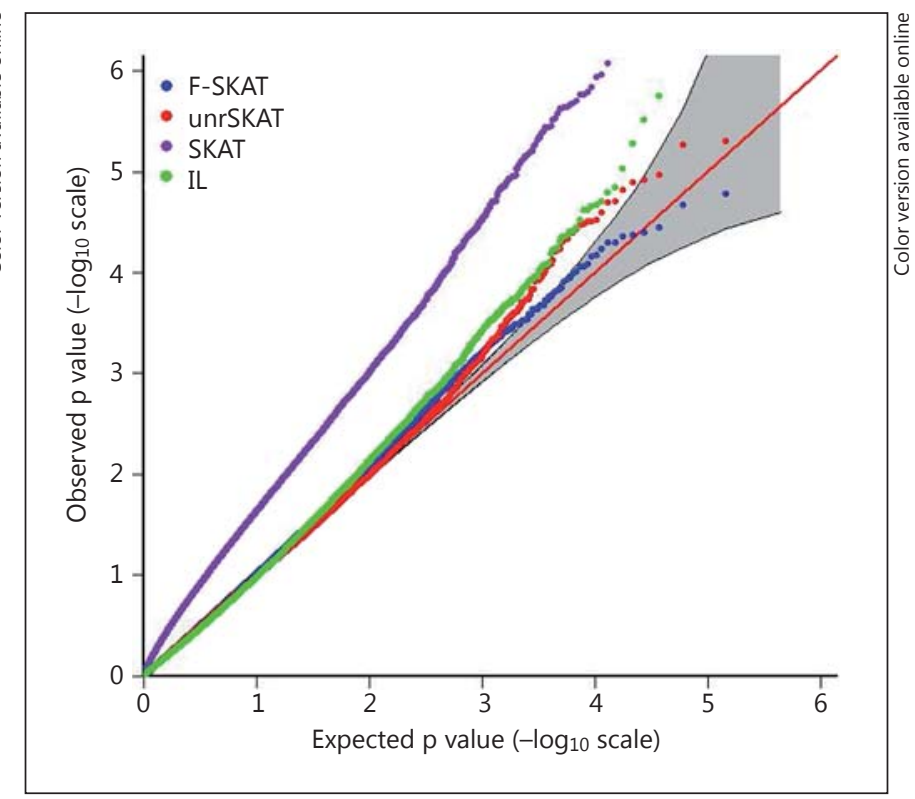

Fig. 4. QQ plot of the p values for F-SKAT, unrSKAT, SKAT, and IL for families with 3 generations from the null simulation, with a 95\% pointwise confidence band (gray area) that is computed under the assumption of the $\mathrm{p}$ values being drawn independently from a uniform $[0,1]$ distribution.

is higher in families with flexible numbers of offspring than in family trios, which indicates that the power gain of F-SKAT increases as the size of families increases. This is expected because F-SKAT makes full use of the data, while in contrast, unrSKAT uses only unrelated samples and discards other family members.

\section{Discussion}

In this paper, we have proposed a new method, FSKAT, under a generalized linear mixed-model framework that can be used to analyze family data with various phenotypes, such as continuous and discrete, and covariates. The new method is based on a kernel machine regression and can be viewed as an extension of SKAT [9, 21] as well as famSKAT [29]. As a set-based analysis, FSKAT shares the advantages of set-based methods, such as improved power by testing a set of genetic variants jointly and by reducing the multiple-testing penalty. Our simulation studies show that the proposed method has consistently higher power than existing approaches in the scenarios we have considered. The new method includes various existing methods, such as SKAT and famSKAT, 
Fig. 5. Power comparisons of F-SKAT, unrSKAT, and IL for data with parent-offspring family trios ( $a$ level ranges from 0 to 0.05 ). a $40 \%$ disease variants scenario; b $80 \%$ disease variants scenario; c $30 \%$ disease variants and $10 \%$ protective variants scenario; d $60 \%$ disease variants and $20 \%$ protective variants scenario.

Fig. 6. Power comparisons of F-SKAT, unrSKAT, and IL for data of families with flexible numbers of offspring (a level ranges from 0 to 0.05 ). a $40 \%$ disease variants scenario; b $80 \%$ disease variants scenario; c $30 \%$ disease variants and $10 \%$ protective variants scenario; d $60 \%$ disease variants and $20 \%$ protective variants scenario.
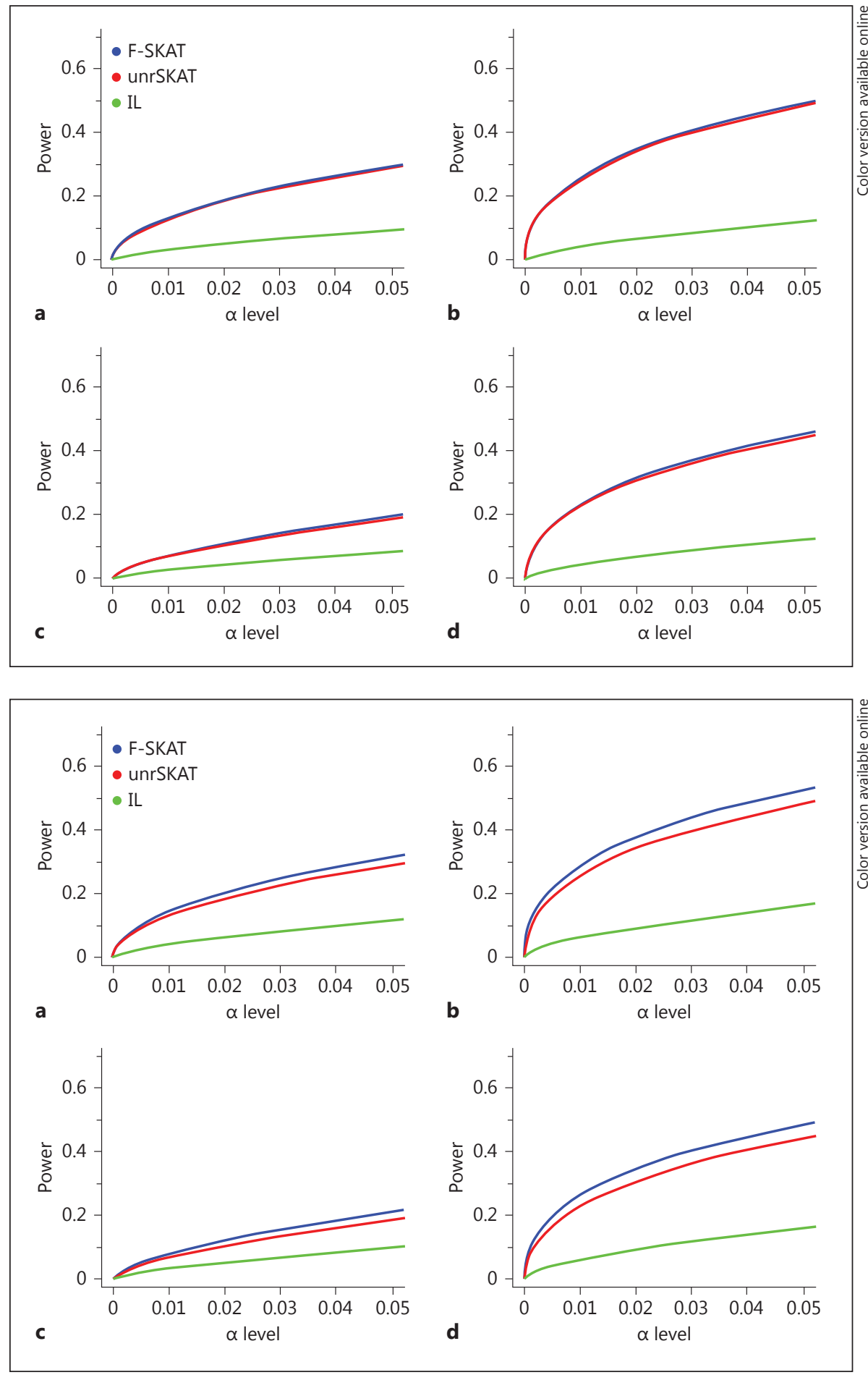

as special cases. This shows that the proposed method is theoretically more advantageous than the existing methods and allows us to conveniently analyze data using different approaches.

SKAT for Dichotomous Traits in Family Samples
In the simulation studies, we show that using SKAT on data with related samples results in an inflated type I error rate, which is consistent with the results for quantitative traits in Chen et al. [29]. One strategy is to analyze only 
Fig. 7. Power comparisons of F-SKAT, unrSKAT, and IL for data of families with 3 generations ( $a$ level ranges from 0 to 0.05 ). a $40 \%$ disease variants scenario; b $80 \%$ disease variants scenario; c $30 \%$ disease variants and $10 \%$ protective variants scenario; d $60 \%$ disease variants and $20 \%$ protective variants scenario.

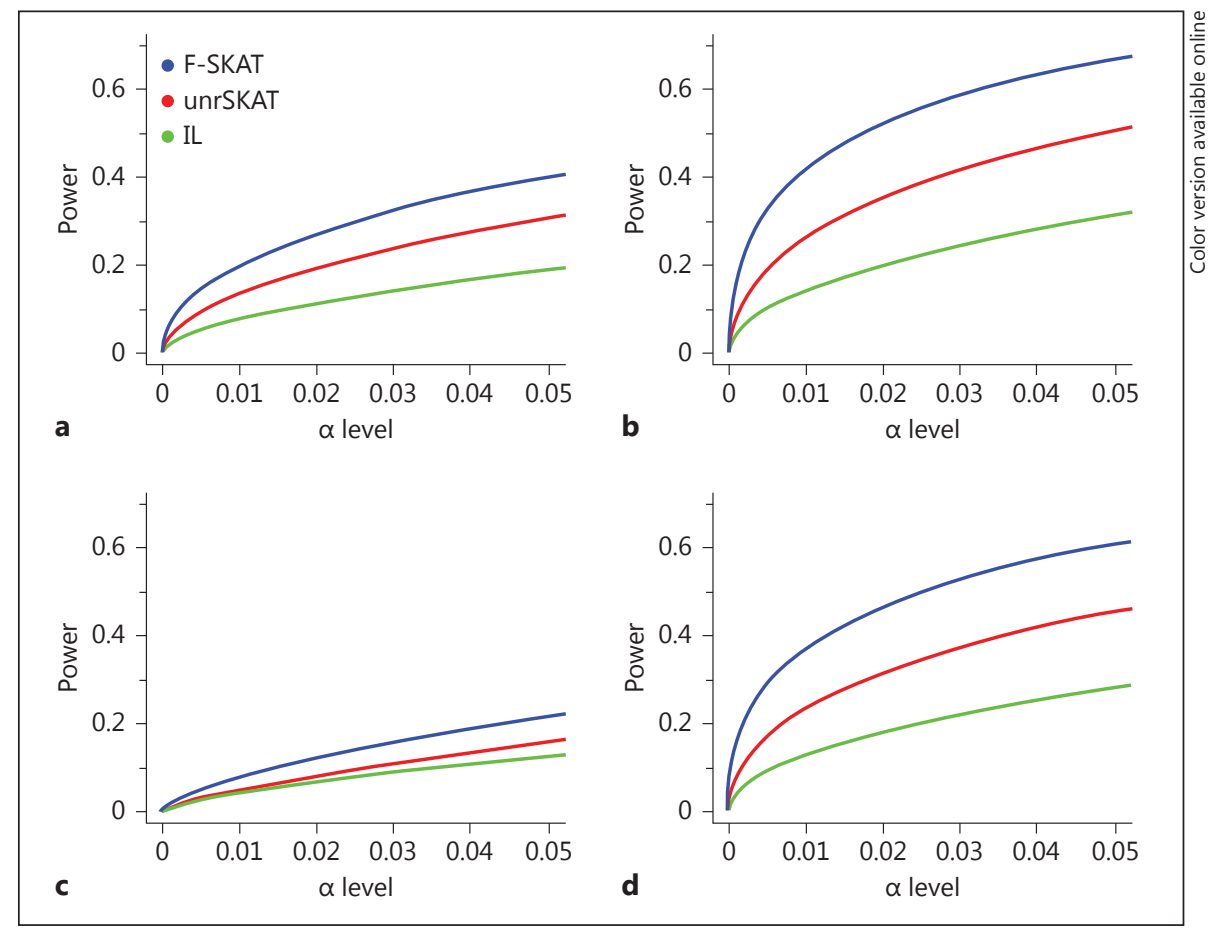

unrelated subjects from the data using SKAT. In this way, the type I error rate can be controlled, but power is sacrificed because only part of the data is analyzed. In contrast, F-SKAT uses all of the data, retains the correct type I error rate, and achieves higher power in all of the scenarios we considered. Our simulation also shows that the larger the family size, the more power F-SKAT gains, which is also consistent with the findings for quantitative traits in Chen et al. [29]. Based on our simulation study, F-SKAT makes a good choice for analysis of family data with various traits, although we only considered dichotomous traits in this study. For quantitative traits, an extensive simulation study has been published by Chen et al. [29].

The computation time of F-SKAT depends on both sample size (including family size and structure) and the number of genetic variants to be analyzed. F-SKAT involves fitting a generalized linear mixed model that must be done iteratively. This consumes much of the computation time. Even though this step is computationally intensive, the total computation time, in fact, may not be a serious issue when analyzing data from GWASs. Like SKAT, F-SKAT is basically a score test and, thus, under $H_{0}$, the estimates of covariate coefficients and covariance matrix are independent with genetic variants. The generalized linear mixed model under $H_{0}$ only needs to be fitted once for the whole genome and is then reused in the anal- ysis of other genes. Therefore, the total computation time is greatly reduced. To estimate the computation time of the proposed methods, we conducted a simple simulation in $\mathrm{R}$ on a single computing node with a $3-\mathrm{GHz} \mathrm{CPU}$ and 4 GB memory, analyzing a 200 -kb region on 1,500 individuals (500 trios). F-STAT needed $5.988 \mathrm{~s}$ for the analysis. Based on this simulation, we can estimate that it may take F-STAT approximately $25 \mathrm{~h}$ to analyze the whole genome ( $3 \mathrm{~Gb})$ on the same samples. Using a computer cluster with multiple nodes, we anticipate that most of the genome-wide data analysis should be finished within hours using the proposed methods.

Thus, complicated generalized linear mixed models can be implemented for GWASs without suffering from a huge amount of computation time. However, if the number of markers in a gene is large, inverting the large matrix is still computationally intensive. One way to handle this would be to partition the markers into smaller groups, such as groups of nonsynonymous or synonymous coding variants. Another way would be to use fast algorithms, such as those implemented in the software EMMA/EMMAX [40, 41], to make our algorithm faster and more efficient. The approach of clustering samples implemented in TASSEL [41] applies naturally to family data. Recently, several new fast algorithms have been proposed for mixed model [42-45]. Some of the new ideas 
may be used in our algorithm. The F-SKAT algorithm has been implemented in R (http://www.r-project.org/) and the source code is available online (http://www.soph.uab. edu/ssg/software).

In our work, the kinship coefficients in the kinship matrices were obtained from family relationships. If genome-wide genotype data are available, it is more advantageous to use genetic markers to estimate the kinship coefficients among individuals [40,46-50]. The use of kinship coefficients enables our method to be applicable to data with any relationship (such as grandparentsgrandchildren and uncles-nephews) and even relationships with cryptic relatedness. We have shown that our new method is feasible for genome-wide studies, although the computation time is still intensive. Fast algorithms, such as those developed for linear mixed models [31, 40, 41, 43-45], are attractive and would be very helpful. Although we have only studied the performance of a linear kernel in this work, it is straightforward to use a non-linear kernel within the flexible kernel machine-regression framework when a non-linear association between a disease and genetic variants is assumed.

\section{Acknowledgments}

This work was supported in part by grant EPS1158862 from the National Science Foundation (to Q.Y., H.K.T.); grants GM081488, R01HL092173, P60AR064172 (to N.L.), 5R01GM069430-08 (to N.Y.), 5R01DA025095 (to X.-Y.L.), and GM073766 (to G.G.) from the National Institutes of Health; grants 102-2628-B-002-039MY3 from the Ministry of Science and Technology of Taiwan and NTU-CESRP-104R7622-8 from National Taiwan University (to W.-Y.L.). The content of this paper is solely the responsibility of the authors and does not necessarily represent the official views of the sponsors. The authors also declare that they have no conflicts of interest.

\section{References}

$\checkmark 1$ Mardis ER: The impact of next-generation sequencing technology on genetics. Trends Genet 2008;24:133-141.

2 Metzker ML: Sequencing technologies - the next generation. Nat Rev Genet 2010;11:3146.

3 Wellcome Trust Case Control Consortium: Genome-wide association study of 14,000 cases of seven common diseases and 3,000 shared controls. Nature 2007;447:661-678.

4 Hunter DJ, Kraft P, Jacobs KB, et al: A genome-wide association study identifies alleles in FGFR2 associated with risk of sporadic postmenopausal breast cancer. Nat Genet 2007;39:870-874

5 Yeager M, Orr N, Hayes RB, et al: Genomewide association study of prostate cancer identifies a second risk locus at $8 \mathrm{q} 24$. Nat Genet 2007;39:645-649.

-6 Hindorff LA, Sethupathy P, Junkins HA, Ramos EM, Mehta JP, Collins FS, Manolio TA: Potential etiologic and functional implications of genome-wide association loci for human diseases and traits. Proc Natl Acad Sci USA 2009; 106:9362-9367.

7 Manolio TA, Brooks LD, Collins FS: A HapMap harvest of insights into the genetics of common disease. J Clin Invest 2008;118: 1590-1605.

8 Liu JZ, McRae AF, Nyholt DR, Medland SE, Wray NR, Brown KM; AMFS Investigators, Hayward NK, Montgomery GW, Visscher PM, Martin NG, Macgregor S: A versatile gene-based test for genome-wide association studies. Am J Hum Genet 2010;87:139-145.
9 Wu MC, Kraft P, Epstein MP, Taylor DM, Chanock SJ, Hunter DJ, Lin X: Powerful SNPset analysis for case-control genome-wide association studies. Am J Hum Genet 2010;86: 929-942.

10 Neale BM, Sham PC: The future of association studies: gene-based analysis and replication. Am J Hum Genet 2004;75:353-362.

-11 Han F, Pan W: A data-adaptive sum test for disease association with multiple common or rare variants. Hum Hered 2010;70:42-54.

12 Hoffmann TJ, Marini NJ, Witte JS: Comprehensive approach to analyzing rare genetic variants. PLoS One 2010;5:e13584.

$\checkmark 13$ Li B, Leal SM: Methods for detecting associations with rare variants for common diseases: application to analysis of sequence data. Am J Hum Genet 2008;83:311-321.

14 Lin WY, Lou XY, Gao G, Liu N: Rare variant association testing by adaptive combination of p-values. PLoS One 2014;9:e85728.

-15 Lin WY, Yi N, Lou XY, Zhi D, Zhang K, Gao G, Tiwari HK, Liu N: Haplotype kernel association test as a powerful method to identify chromosomal regions harboring uncommon causal variants. Genet Epidemiol 2013;37: 560-570.

16 Lin WY, Yi N, Zhi D, Zhang K, Gao G, Tiwari HK, Liu N: Haplotype-based methods for detecting uncommon causal variants with common SNPs. Genet Epidemiol 2012;36:572582.

17 Madsen BE, Browning SR: A groupwise association test for rare mutations using a weighted sum statistic. PLoS Genet 2009;5:e1000384.
18 Morgenthaler S, Thilly WG: A strategy to discover genes that carry multi-allelic or monoallelic risk for common diseases: a cohort allelic sums test (CAST). Mutat Res 2007;615: 28-56.

19 Zawistowski M, Gopalakrishnan S, Ding J, Li Y, Grimm S, Zollner S: Extending rare-variant testing strategies: analysis of noncoding sequence and imputed genotypes. Am J Hum Genet 2010;87:604-617.

20 Yi N, Liu N, Zhi D, Li J: Hierarchical generalized linear models for multiple groups of rare and common variants: jointly estimating group and individual-variant effects. PLoS Genet 2011;7:e1002382.

21 Wu MC, Lee S, Cai T, Li Y, Boehnke M, Lin $\mathrm{X}$ : Rare-variant association testing for sequencing data with the sequence kernel association test. Am J Hum Genet 2011;89:82-93.

22 Yan Q, Tiwari HK, Yi N, Lin WY, Gao G, Lou XY, Cui X, Liu N: Kernel-machine testing coupled with a rank-truncation method for genetic pathway analysis. Genet Epidemiol 2014;38:447-456.

23 Falk CT, Rubinstein P: Haplotype relative risks: an easy reliable way to construct a proper control sample for risk calculations. Ann Hum Genet 1987;51:227-233.

24 Ott J: Statistical properties of the haplotype relative risk. Genet Epidemiol 1989;6:127130.

-25 Spielman RS, McGinnis RE, Ewens WJ: Transmission test for linkage disequilibrium: the insulin gene region and insulin-dependent diabetes mellitus (IDDM). Am J Hum Genet 1993;52:506-516. 
26 Terwilliger JD, Ott J: A haplotype-based 'haplotype relative risk' approach to detecting allelic associations. Hum Hered 1992;42:337346.

27 Almasy L, Blangero J: Multipoint quantitative-trait linkage analysis in general pedigrees. Am J Hum Genet 1998;62:1198-1211.

28 Rabinowitz D, Laird N: A unified approach to adjusting association tests for population admixture with arbitrary pedigree structure and arbitrary missing marker information. Hum Hered 2000;50:211-223.

29 Chen H, Meigs JB, Dupuis J: Sequence kernel association test for quantitative traits in family samples. Genet Epidemiol 2013;37:196204.

-30 Schifano ED, Epstein MP, Bielak LF, Jhun MA, Kardia SL, Peyser PA, Lin X: SNP set association analysis for familial data. Genet Epidemiol 2012;36:797-810.

31 Oualkacha K, Dastani Z, Li R, Cingolani PE Spector TD, Hammond CJ, Richards JB, Ciampi A, Greenwood CM: Adjusted sequence kernel association test for rare variants controlling for cryptic and family relatedness. Genet Epidemiol 2013;37:366-376.

32 Ionita-Laza I, Lee S, Makarov V, Buxbaum JD, Lin X: Family-based association tests for sequence data, and comparisons with population-based association tests. Eur J Hum Genet 2013;21:1158-1162.

33 Cordell HJ: Summary of results and discussions from the gene-based tests group at genetic analysis workshop 18. Genet Epidemiol 2014;38(suppl 1):S44-S48.
34 Kwee LC, Liu D, Lin X, Ghosh D, Epstein MP: A powerful and flexible multilocus association test for quantitative traits. Am J Hum Genet 2008;82:386-397.

35 Liu D, Lin X, Ghosh D: Semiparametric regression of multidimensional genetic pathway data: least-squares kernel machines and linear mixed models. Biometrics 2007;63: 1079-1088.

36 Zhang D, Lin X: Hypothesis testing in semiparametric additive mixed models. Biostatistics 2003;4:57-74.

37 Yuan KH, Bentler PM: Two simple approximations to the distributions of quadratic forms. Br J Math Stat Psychol 2010;63:273291.

38 Davies R: The distribution of a linear combination of chi-square random variables. J $\mathrm{R}$ Stat Soc Ser C Appl Stat 1980;29:323-333.

-39 Schaffner SF, Foo C, Gabriel S, Reich D, Daly MJ, Altshuler D: Calibrating a coalescent simulation of human genome sequence variation. Genome Res 2005;15:1576-1583.

40 Kang HM, Sul JH, Service SK, Zaitlen NA, Kong SY, Freimer NB, Sabatti C, Eskin E: Variance component model to account for sample structure in genome-wide association studies. Nat Genet 2010;42:348-354.

41 Zhang Z, Ersoz E, Lai CQ, Todhunter RJ, Tiwari HK, Gore MA, Bradbury PJ, Yu J, Arnett DK, Ordovas JM, Buckler ES: Mixed linear model approach adapted for genome-wide association studies. Nat Genet 2010;42:355360.

42 Zhou X, Stephens M: Efficient multivariate linear mixed model algorithms for genomewide association studies. Nat Methods 2014; 11:407-409.
43 Lippert C, Listgarten J, Liu Y, Kadie CM, Davidson RI, Heckerman D: Fast linear mixed models for genome-wide association studies. Nat Methods 2011;8:833-835.

44 Svishcheva GR, Axenovich TI, Belonogova NM, van Duijn CM, Aulchenko YS: Rapid variance components-based method for whole-genome association analysis. Nat $\mathrm{Ge}$ net 2012;44:1166-1170.

45 Zhou X, Stephens M: Genome-wide efficient mixed-model analysis for association studies. Nat Genet 2012;44:821-824.

46 Balding DJ, Nichols RA: A method for quantifying differentiation between populations at multi-allelic loci and its implications for investigating identity and paternity. Genetica 1995;96:3-12.

47 Lynch M, Ritland K: Estimation of pairwise relatedness with molecular markers. Genetics 1999;152:1753-1766.

48 Ritland K: Multilocus estimation of pairwise relatedness with dominant markers. Mol Ecol 2005;14:3157-3165.

49 Yu J, Pressoir G, Briggs WH, Vroh Bi I, Yamasaki M, Doebley JF, McMullen MD, Gaut BS, Nielsen DM, Holland JB, Kresovich S, Buckler ES: A unified mixed-model method for association mapping that accounts for multiple levels of relatedness. Nat Genet 2006; 38:203-208.

50 Liu N, Zhao H, Patki A, Limdi NA, Allison DB: Controlling population structure in human genetic association studies with samples of unrelated individuals. Stat Interface 2011; 4:317-326. 